\title{
L'influence des procédés technologiques de préparation du lait de consommation sur la teneur en pesticides organochlorés
}

\author{
par \\ R. VAN RENTERGHEM \\ Station Laitière de l'Etat, Melle \\ Centre de Recherches Agronomiques de l'Etat, Gand (Belgique)
}

\section{INTRODUCTION}

On dispose d'un certain nombre de données relatives à l'éliminaticn des pesticides organochlorés pendant les traitements industriels et ménagers des légumes et des fruits (Farrow et al., 1966 ; Hemphill et al., 1967 ; Elkins et al., 1968 ; Farrow et al., 1968 ; Lamb et al., 1968).

Les données analogues relatives au lait et aux produits laitiers sont moins nombreuses.

Quoique l'approche la plus logique de ce problème de résidus consiste à prévenir la contamination à la ferme, un examen de toutes les méthodes susceptibles de diminuer la teneur en pesticides organochlorés du lait et des produits laitiers reste souhaitable.

Nous avons étudié l'influence de la technologie de la préparation du lait de consommation.

\section{APERÇU BIBLIOGRAPHIQUE}

Les pesticides organochlorés présents en tant que résidus dans le lait et les produits laitiers s'y trouvent dans la phase grasse. Ils y manifestent une certaine affinité pour la fraction lipoprotéinique.

De nombreux chercheurs, tels que Langlois et al. (1964, 1965), Bills et Sloan (1967), Ledford et al. (1968), Li et Bradley (1969) et Hekmati et Bradley (1971) ont augmenté artificiellement la teneur en pesticides du lait à examiner, mais Kroger (1968) et Ledford et 


\section{TABLEAU 1}

Données bibliographiques relatives à l'influence de la pasteurisation et de la stérilisation sur la teneur en pesticides organochlorés du lait

\begin{tabular}{|c|c|c|c|c|c|}
\hline \multirow{2}{*}{ Procédé } & \multirow{2}{*}{ Pesticide } & \multicolumn{2}{|c|}{$\begin{array}{l}\text { Teneur en pesticide } \\
\text { en p.p.m. / } \\
\text { matière grasse }\end{array}$} & \multirow{2}{*}{$\begin{array}{c}\text { Modification } \\
\text { du taux } \\
\text { de } \\
\text { pesticides } \\
\text { en p. } 100\end{array}$} & \multirow{2}{*}{ Références } \\
\hline & & $\begin{array}{c}\text { avant le } \\
\text { traitement }\end{array}$ & $\begin{array}{c}\text { après le } \\
\text { traitement }\end{array}$ & & \\
\hline Pasteurisation & DDT & 150 & 120 & -20 p. 100 & Mann et al., 1950 \\
\hline $\begin{array}{l}\text { Pasteurisation } \\
30 \mathrm{mn} 62,8^{\circ} \mathrm{C}\end{array}$ & $\begin{array}{c}\text { DDT } \\
\text { lindane }\end{array}$ & $\begin{array}{l}26,47 \\
25,13\end{array}$ & $\begin{array}{l}25,59 \\
20,51\end{array}$ & $\begin{array}{r}3,3 \text { p. } 100 \\
-18,4 \text { p. } 100\end{array}$ & Langlois et al., 1964 \\
\hline $\begin{array}{l}\text { Pasteurisation } \\
30 \mathrm{mn} 62,8^{\circ} \mathrm{C}\end{array}$ & dieldrine & 26,14 & 26,80 & $+2,5$ p. 100 & Langlois et al., 1965 \\
\hline $\begin{array}{l}\text { Pasteurisation } \\
30 \mathrm{mn} 63^{\circ} \mathrm{C}\end{array}$ & $\begin{array}{c}\text { lindane } \\
\text { lindane } \\
\text { dieldrine } \\
\text { dieldrine } \\
\text { hepo } \\
\text { hepo } \\
\text { DDE } \\
\text { DDE } \\
\text { DDD } \\
\text { DDD } \\
\text { DDT } \\
\text { DDT }\end{array}$ & $\begin{array}{l}0,21 \\
0,23 \\
4,13 \\
3,16 \\
0,12 \\
0,15 \\
0,12 \\
0,19 \\
0,38 \\
0,47 \\
0,21 \\
0,23\end{array}$ & $\begin{array}{l}0,22 \\
0,22 \\
4,04 \\
3,13 \\
0,13 \\
0,15 \\
0,13 \\
0,20 \\
0,37 \\
0,46 \\
0,21 \\
0,25\end{array}$ & $\begin{array}{c}+\quad 4,8 \text { p. } 100 \\
-\quad 4,3 \text { p. } 100 \\
-\quad 2,2 \text { p. } 100 \\
-\quad 0,9 \text { p. } 100 \\
+\quad 8,3 \text { p. } 100 \\
0 \\
+\quad 8,3 \text { p. } 100 \\
+\quad 5,3 \text { p. } 100 \\
-\quad 2,6 \text { p. } 100 \\
-\quad 2,1 \text { p. } 100 \\
0 \\
+\quad 8,7 \text { p. } 100\end{array}$ & Li et al., 1970 \\
\hline $\begin{array}{l}\text { Stérilisation de } \\
\text { lait condensé } \\
15 \mathrm{mn} \\
115,5-118,3^{\circ} \mathrm{C}\end{array}$ & \begin{tabular}{|} 
lindane \\
lindane \\
dieldrine \\
dieldrine \\
hepo \\
hepo \\
DDE \\
DDE \\
DDD \\
DDD \\
DDT \\
DDT
\end{tabular} & $\begin{array}{l}0,09 \\
0,09 \\
2,20 \\
2,37 \\
0,15 \\
0,13 \\
0,19 \\
0,17 \\
0,23 \\
0,21 \\
0,20 \\
0,17\end{array}$ & $\begin{array}{l}0,07 \\
0,06 \\
2,20 \\
2,37 \\
0,11 \\
0,12 \\
0,17 \\
0,16 \\
0,20 \\
0,19 \\
0,15 \\
0,12\end{array}$ & $\begin{array}{r}-22,2 \text { p. } 100 \\
-33,3 \text { p. } 100 \\
0 \\
0 \\
-26,7 \text { p. } 100 \\
-7,7 \text { p. } 100 \\
-10,5 \text { p. } 100 \\
-5,9 \text { p. } 100 \\
-13,0 \text { p. } 100 \\
-9,5 \text { p. } 100 \\
-25,0 \text { p. } 100 \\
-29,4 \text { p. } 100\end{array}$ & \\
\hline $\begin{array}{l}\text { Stérilisation de } \\
\text { lait condensé } \\
15 \mathrm{mn} 115,5^{\circ} \mathrm{C}\end{array}$ & $\begin{array}{c}\text { DDT } \\
\text { lindane }\end{array}$ & $\begin{array}{l}23,05 \\
26,42\end{array}$ & $\begin{array}{l}25,12 \\
25,63\end{array}$ & $\begin{array}{l}+\quad 9,0 \text { p. } 100 \\
-\quad 3,0 \text { p. } 100\end{array}$ & Langlois et al., 1964 \\
\hline $\begin{array}{l}\text { Stérilisation de } \\
\text { lait condensé } \\
15 \mathrm{mn} 115,5^{\circ} \mathrm{C}\end{array}$ & $\begin{array}{c}\text { dieldrine } \\
\text { hepo }\end{array}$ & $\begin{array}{l}11,38 \\
10,96\end{array}$ & $\begin{array}{l}11,19 \\
12,56\end{array}$ & $\begin{array}{l}1,7 \text { p. } 100 \\
+14,6 \text { p. } 100\end{array}$ & Langlois et al., 1965 \\
\hline
\end{tabular}




\section{TABLEAU 1 (suite)}

\begin{tabular}{|c|c|c|c|c|c|}
\hline \multirow{2}{*}{ Procédé } & \multirow{2}{*}{ Pesticide } & \multicolumn{2}{|c|}{$\begin{array}{l}\text { Teneur en pesticide } \\
\text { en p.p.m./ } \\
\text { matière grasse }\end{array}$} & \multirow{2}{*}{ 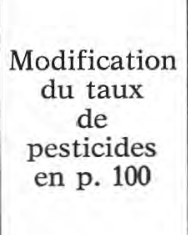 } & \multirow{2}{*}{ Références } \\
\hline & & $\begin{array}{l}\text { avant le } \\
\text { traitement }\end{array}$ & $\begin{array}{c}\text { après le } \\
\text { traitement }\end{array}$ & & \\
\hline $\begin{array}{l}\text { Stérilisation de } \\
\text { lait condensé } \\
15 \mathrm{mn} 115,5^{\circ} \mathrm{C}\end{array}$ & $\begin{array}{c}\text { lindane } \\
\text { dieldrine } \\
\text { hepo } \\
\text { DDT }\end{array}$ & $\begin{array}{l}26,4 \\
11,4 \\
11,0 \\
23,1\end{array}$ & $\begin{array}{l}25,6 \\
11,2 \\
12,6 \\
25,1\end{array}$ & $\begin{array}{r}3,0 \text { p. } 100 \\
-\quad 1,8 \text { p. } 100 \\
+\quad 14,5 \text { p. } 100 \\
+\quad 8,7 \text { p. } 100\end{array}$ & Liska, 1968 \\
\hline $\begin{array}{l}\text { Stérilisation de } \\
\text { lait condensé } \\
15 \mathrm{mn} 115,5^{\circ} \mathrm{C}\end{array}$ & DDT & - & - & shift au DDD & Langlois et al., 1964 \\
\hline
\end{tabular}

al. (1968) ont déjà signalé que les pesticides ainsi ajoutés se comportent autrement que ceux incorporés par des voies physiologiques. Les diminutions de teneurs doivent dès lors être considérées comme maximales dans des recherches de ce genre. Downey (1973) fait remarquer en outre que l'extractibilité des pesticides peut être tellement influencée par les procédés technologiques mis en œuvre, que leur élimination est, en partie, plus apparente que réelle. Nous n'avons utilisé pour nos recherches que des échantillons de lait contaminés par la voie physiologique.

Mannetal. (1950), Langlois et al. (1964, 1965), Liska (1968) et Li et al. (1970) ont déjà étudié pour un nombre limité de composés organochlorés présents dans le lait l'influence que la pasteurisation et la stérilisation exercent sur la teneur en ces produits. Le tableau 1 donne un aperçu des résultats obtenus par ces chercheurs. On ne peut pas en déduire que la pasteurisation et la stérilisation font baisser notablement la teneur en pesticides organochlorés du lait.

Stempet Liska (1966), Mc Caskey et Liska (1967), Liska (1968) et Liet al. (1970) ont constaté, après stérilisation, d'importantes diminutions de teneurs en certains composés organochlorés moins importants tels que le dicofol, l'endosulfansulfate, le chlordane et le telodrin.

La bibliographie ne fournit aucune donnée relative à l'influence de l'upérisation. 


\section{MATERIEL ET METHODES}

\subsection{Pesticides étudiés}

Nos recherches ont porté sur les pesticides organochlorés suivants : l'hexachlorobenzène (HCB), l' $\alpha$-hexachlorocyclohexane $(\alpha \mathrm{HCH}$ ou $\alpha \mathrm{BHC})$, le $\beta$-hexachlorocyclohexane $(\beta \mathrm{HCH}$ ou $\beta \mathrm{BHC})$, le $\gamma$-hexachlorocyclohexane $(\gamma \mathrm{HCH}$ ou $\gamma \mathrm{BHC}$ ou lindane), l'heptachlore-époxyde, la dieldrine, le ppDDT, l'opDDT, le ppDDE et le ppDDD (ppTDE).

\subsection{Traitement du lait de consommation}

\subsubsection{Pasteurisation}

Chaque fois dix échantillons ont été prélevés sur trois lots différents de lait de mélange (3,2 p. 100 de matière grasse) avant et après la pasteurisation pendant $15 \mathrm{~s}$ à $73^{\circ} \mathrm{C}$. Le lait pasteurisé n'a pas été homogénéisé.

\subsubsection{STÉRILISATION}

Chaque fois cinq échantillons de lait (3,2 p. 100 de matière grasse) ont été prélevés sur neuf productions différentes avant et après la stérilisation dans des installations industrielles. La pré-stérilisation s'effectuait à $137^{\circ} \mathrm{C}$ pendant $10 \mathrm{~s}$ et la post-stérilisation à $118^{\circ} \mathrm{C}$ pendant $10 \mathrm{mn} 30 \mathrm{~s}$. Le lait était homogénéisé après la pré-stérilisation.

\subsubsection{UPÉRISATION}

Chaque fois cinq échantillons de lait (3,2 p. 100 de matière grasse) ont été prélevés sur neuf productions différentes à injection directe de vapeur, avant et après l'upérisation à $150^{\circ} \mathrm{C}$ pendant $2,4 \mathrm{~s}$. Le lait upérisé a été homogénéisé.

\subsection{Méthode d'analyse}

La détermination des pesticides organochlorés présents dans le lait a été faite selon la méthode « single step » de Holden et Marsden (1969) adaptée pour les échantillons de lait. $50 \mathrm{ml}$ de lait ont été additionnés de $100 \mathrm{ml}$ d'acétone. Après mélange avec $100 \mathrm{ml}$ de pentane et séparation des phases, la couche de pentane contenant la matière grasse a été filtrée sur du sulfate de sodium anhydre, puis évaporée. $200 \mathrm{mg}$ de la phase grasse ainsi obtenue et contenant les pesticides ont été transférés sur une colonne d'oxyde d'aluminium (basique, désactivé de $10 \mathrm{p} .100$ ) et élués avec $80 \mathrm{ml}$ de pentane. Après concentration de l'éluat à $1 \mathrm{ml}$, par évaporation, 3 à $7 \mu \mathrm{l}$ en ont été injectés dans un chromatographe en phase gazeuse avec détecteur de capture d'électrons. Les colonnes étaient chargées de 
respectivement 3 p. 100 OV $17 /$ OV $210: 3 / 22$ et 1,5 p. 100 OV $17 / 1,95$ p. $100 \mathrm{QF}_{1}$ : 1/13, dans les deux cas sur Gaschrom Q, 80-100 mesh.

\section{RESULTATS ET COMMENTAIRES}

L'erffet que les différents traitements du lait de consommation exercent sur la teneur en pesticides organochlorés a été examiné par application de l'épreuve du $\mathrm{t}$ aux résultats d'analyse.

Les déterminations des différents pesticides, faites avant et après le traitement, pouvant être considérées comme des observations par paires, l'épreuve du $t$ a été appliquée à la différence moyenne, ce qui éliminait la variabilité entre échantillons et la variabilité entre productions. L'épreuve a été appliquée bilatéralement puisque l'on ne pouvait pas poser a priori que le procédé technologique entraîne un déplacement dans un sens déterminé de la teneur en pesticides. Le tableau 2 indique, pour chacune des trois sortes de lait de consommation, la teneur moyenne avant et après le traitement, la différence moyenne, la modification en p.100, l'écart standard par rapport à la différence moyenne et la valeur t calculée pour les différents pesticides. Il indique aussi, de façon conventionnelle, si cette valeur t calculée est significative à $\alpha=0,05\left(^{*}\right)$ ou à $\left.\alpha=0,01{ }^{* *}\right)$.

Les différences moyennes entre les teneurs avant et celles après le traitement ont été tantôt positives, tantôt négatives. Dans le cas de la pasteurisation elles sont principalement négatives. Les différences sont cependant minimes; elles ne sont significatives à $\alpha=0,05$ que dans le cas du ppDDE, de la dieldrine et du ppDDT. Dans le cas du $\beta \mathrm{HCH}$, par contre, on relève une différence positive significative à $\alpha=0,05$.

Sauf pour l'heptachlore-époxyde, les moyennes sont plus élevées après la stérilisation qu'avant, tout en n'étant significatives à $\alpha=0,05$ que dans le cas du HCB.

Après upérisation, des différences significatives à $\alpha=0,01$ sont à relever pour cinq pesticides, en l'occurrence le $\mathrm{HCB}$, l'heptachloreepoxyde, le ppDDE, la dieldrine et le ppDDT. Les différences sont négatives, mais non significatives dans le cas du $\gamma \mathrm{HCH}$ et du ppDDD.

A remarquer que le lait stérilisé et le lait upérisé ont été homogénéisés et que le lait pasteurisé n'a pas subi ce traitement.

Il est apparu que le traitement thermique et l'homogénéisation appliqués au cours de la préparation du lait stérilisé et du lait upérisé augmentent l'extractibilité des pesticides organochlorés incorporés par la voie physiologique lorsque l'extraction est effectuée à l'acétone-pentane. Cette augmentation est plus considérable pour le lait upérisé que pour le lait stérilisé. Cet effet est soit imputable au 
TABLEAU 2

Influence de la pasteurisation, de la stérilisation et de l'u périsation sur la teneur en pesticides organochlorés du lait

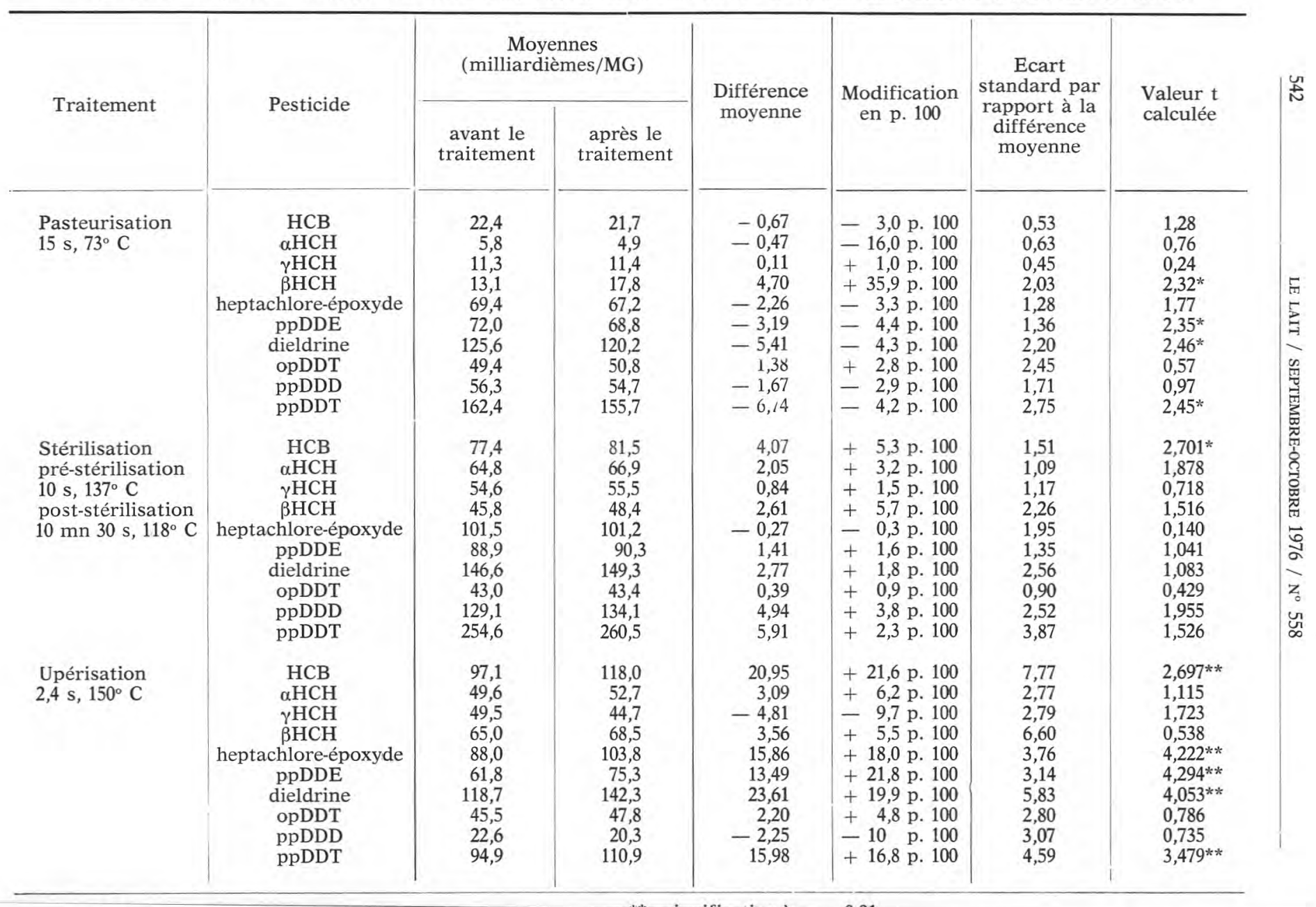


degré d'homogénéisation soit proportionnel à la température d'application du traitement. L'influence de l'homogénéisation sur l'extractibilité est explicable si l'on prend en considération l'affinité des pesticides organochlorés pour les lipoprotéines de la membrane du globule de graisse. La conséquence de la modification d'extractibilité est une augmentation apparente de la teneur en pesticides organochlorés lorsque l'extraction est effectuée à l'acétone-pentane.

La diminution (non significative) de la teneur en lindane $(\gamma \mathrm{HCH})$ dans le cas du lait upérisé peut s'expliquer si l'on admet que ce composant relativement volatil disparaît effectivement. Une destruction des pesticides organochlorés est peu probable aux températures mises en œuvre.

\section{v. CONCLUSIONS}

Les recherches décrites ci-dessus n'ont pas permis de conclure à une diminution significative de la teneur en pesticides organochlorés au cours de la transformation en lait de consommation de laits contaminés par la voie physiologique. Ceci concorde avec les données bibliographiques.

L'influence que la température et/ou l'homogénéisation appliquées au cours de la préparation du lait stérilisé ou upérisé exercent sur l'extractibilité à l'acétone-pentane des pesticides organochlorés donne lieu, en outre, à une augmentation apparente de la teneur en ces pesticides. Cette augmentation apparente est toutefois minime (en moyenne 6,5 p. 100).

\section{Remerciements}

L'auteur remercie l'ingénieur $R$. Moermans, du «Bureau de Biométrie " du Centre de Recherches Agronomiques de l'Etat, Gand, ainsi que MM. A. Bassier et $T$. Wauters, ingénieurs-techniciens, de leur précieuse collaboration.

\section{Rés u mé}

L'auteur étudie l'influence des procédés technologiques de traitement du lait de consommation (pasteurisation, stérilisation, upérisation) sur la teneur en pesticides organochlorés de celui-ci.

Il est peu probable que les températures utilisées aient une influence destructive quelconque sur les composés organochlorés. L'élimination de ces composés par les procédés examinés n'est pas significative. Le traitement thermique et l'homogénéisation au cours de la préparation du lait de consommation augmentent dans une faible mesure l'extractibilité des pesticides organochlorés incorporés 
par la voie physiologique lorsque l'extraction est effectuée à l'acétonepentane.

L'affinité de ces composés avec les lipoprotéines de la membrane du globule de graisse et l'influence que l'homogénéisation exerce sur ces membranes pourraient expliquer ce phénomène.

\begin{abstract}
It was investigated to what extent the technological methods used to process market milk (pasteurization, sterilization, uperization) influence its organochlorine pesticide content.

It is hardly likely that the temperatures applied exercise a destructive influence on the organochlorine compounds. The degradation of these compounds, due to the procedures investigated, is not significant. The heat treatment and the homogenizing in processing market milk obviously slightly increase the extractibility of physiologically incorporated organochlorine pesticides, when the extraction is carried out using acetone-pentane.

The affinity of these compounds with the lipoproteins of the fat globule membrane and the influence of homogenizing on these membranes may explain this phenomenon.
\end{abstract}

\title{
Zusammenfassung
}

Es wurde untersucht, inwiefern die Verfahrenstechniken, die bei der Herstellung von Trinkmilch angewandt werden (Pasteurisierung, Sterilisierung, Uperisierung) den Organochlorpestizid-Gehalt beeinflussen.

Es ist wenig wahrscheinlich, dass die verwendeten Temperaturen irgendwelchen vernichtenden Einfluss auf die Organochlorverbindungen ausüben. Der Abbau dieser Verbindungen durch die untersuchten Verfahren ist nicht bedeutsam. Die Wärmebehandlung und die Homogenisierung bei der Herstellung von Trinkmilch erhöhen augenscheinlich in geringem Masse die Extrahierbarkeit physiologisch inkorporierter Organochlorpestizide, wenn die Extraktion mit AcetonPentan vorgenommen wird.

Die Affinität dieser Verbindungen für die Lipoproteide der Fettkugelmembran und der Einfluss der Homogenisierung auf diese Membran können diese Erscheinung erklären. 


\section{Références}

BILLS (D. D.) and SloAN (J. L.) (1967). - Removal of chlorinated insecticide residues from milk fat by molecular distillation. J. Agr. Fd Chem., 15, 676.

DoWNEY (W. K.) (1972). - Pesticide residues in milk and milk products. I.D.F . Annual Bulletin, Part II.

Elkins (E. R.), LaMb (F. C.), Farrow (R. P.), Cook (R. W.), KaWai (M.) and Kimball (J. R.) 1968). - Removal of DDT, malathion and carbaryl from green beans by commercial and home preparative procedures. I. Agr. Fd Chem., 16,962 .

FARRow (R. P.), ElKINS (E. R.), CooK (R. W.) (1966). - Conversion of DDT to TDE in canned spinach. J. Agr. Fd Chem., 14, 430.

Farrow (R. P.), Lamb (F. C.), Cook (R. W.), Kimball (J.R.) and Elkins (E.R.) (1968). - Removal of DDT, malathion and carbaryl from tomatoes by commercial and home preparative methods. J. Agr. Fd Chem., 16, 65.

Hekmati (M.) and BRAdLey (R. L.) (1971). - Degradation of organochlorine pesticides with hydrogen peroxide. Milchwissenschaft, 26, 224.

Hemphill (D. D.), Baldwin (R. E.), Deguzman (A.) and Deloach (H. K.). (1967), Effects of washing, trimming and cooking on levels of DDT and derivatives in green beans. J. Agr. Fd Chem., 15, 290.

HoLden (A. V.) and MARSDEN (K.) (1969). - Single stage clean-up of animal tissue extracts for organochlorine residue analysis. J. Chromatog., 14, 481.

KROGER (M.) (1968). - Effect of various physical treatments on certain organochlorine hydrocarbon insecticides found in milk fat. J. Dairy Sci., 51, 196.

LaMb (F. C.), Farrow (R. P.), Elkins (E. R.), Cook (R. W.) and Kimball (J.R.) 1968). - Behavior of DDT in potatoes during commercial and home preparation. J. Agr. Fd Chem., 16, 272.

Langlois (B. E.), LisKa (B. J.) and Hill (D. L.) (1964). - The effects of processing and storage of dairy products on chlorinated insecticide residues. I. DDT and lindane. J. Milk Fd Technol., 27, 264.

LANGLoIs (B. E.), LisKA (B. J.) and HiLL (D. L.) (1965). - The effects of processing and storage of dairy products on chlorinated insecticide residues. II. Endrin, dieldrin, and heptachlor. J. Milk Fd Technol., 28, 9.

LEDFoRd (R. A.), CHEN (J.H.) and SHIPE (W. F.) (1968). - Effect of direct steam heating and vacuum treatment on levels of pesticide residues in milk. J. Dairy Sci., 51, 219.

Lr (C. F.) and BRADLEY (R. L.) (1969). - Degradation of chlorinated hydrocarbon pesticides in milk and butteroil by ultraviolet energy. J. Dairy Sci., 52, 27.

Li (C. F.), Bradley (R. L.) and Schulte (L. H.) (1970). - Fate of organochlorine pesticides during processing of milk into dairy products. J. A.O.A.C., 53, 127.

LISKA (B. J.) (1968). - Effects of processing on pesticide residues in milk. J. Anim. Sc., 27, 827.

MAC CASKeY (T. A.) and LiSKA (B. J.) (1967). - Effect of milk processing methods on endosulfan, endosulfan sulfate, and chlordane residues in milk. J. Dairy Sci., 50, 1991.

MANN (H.D.), CarTer (R. H.) and Ely (R. E.) (1950). - The DDT content of milk products. J. Milk Fd Technol., 13, 340.

STEMP (A. R.) and LisKA (B. J.) (1966). - Effects of processing and storage of dairy products on telodrin and methoxychlor residues. J. Dairy Sci., 49, 1006. 